\title{
Effect of Test Protocol on Torque Production of the Rotators of the Shoulder
}

\author{
By: Douglas R. Keskula, PhD, PT, ATC and David H. Perrin, PhD, ATC
}

Keskula, D.R., \& Perrin, D.H. (1994). Effect of test protocol on concentric and eccentric torque production of the rotators of the shoulder. Isokinetics and Exercise Science, 4: 176-181.

Made available courtesy of IOS Press: http://www.iospress.nl/loadtop/load.php?isbn=09593020

***Note: Figures may be missing from this format of the document

\begin{abstract}
:
The purpose of this investigation was to determine the effect of test protocol on concentric and eccentric torque production of the rotators of the shoulder. Twenty-eight female subjects were randomly assigned to participate in either a continuous (no pause between concentric and eccentric contractions) or interrupted (5-second pause between contractions) measurement protocol. A Kin-Corn dynamometer (Chattecx Corp., Hixson, TN) was used to obtain average torque measurements at so and $180 \mathrm{deg} / \mathrm{sec}$ for the shoulder rotators. Analysis of variance determined that there were no significant $(\mathrm{p}>0.05)$ differences in average torque between test protocols for either muscle group. Intraclass correlation coefficients (ICCs) ranged from 0.57 to 0.88 for the continuous protocol and from 0.83 to 0.94 for the interrupted protocol. Standard error of measurement estimates ranged from 0.7 to $1.5 \mathrm{Nm}$ for the continuous protocol and from 0.5 to $1.4 \mathrm{Nm}$ for the interrupted protocol. The results of this investigation demonstrate no differences in torque production between continuous and interrupted test protocols, although the interrupted measurement protocol provides greater reliability and precision of measurement than the continuous protocol when assessing torque production of the rotators of the shoulder. Keywords: Rest interval; shoulder internal/external rotation; test reliability

\section{Article:}

\section{INSTRUCTION}

Isokinetic assessment of human muscle performance has become an integral component of clinical practice and research. The use of computer-controlled isokinetic devices provides the clinician/researcher with a means to evaluate and document muscle performance. A variety of test options may be selected based on the goals of the evaluation and capacity of the device to measure isometric, concentric, and eccentric performance of major muscle groups.
\end{abstract}

The Kin-Com dynamometer (Chattecx Corp., Hixson, TN) has been used by a number of investigators to assess concentric and eccentric muscle performance of the elbow, ${ }^{4}$ knee,${ }^{6,9,18,19,20}$ and shoulder. ${ }^{5.8 .10,14}$ The Kin-Com has the capability of assessing concentric-eccentric muscle performance in an interrupted (pause between contractions) or continuous (no pause between contractions).

A time interval spaced between contractions during measurement of muscle performance may serve to decrease the effect of fatigue on subsequent contractions, ${ }^{1,12,17}$ minimize the effect of potentiation, ${ }^{18}$ and reduce the efficiency of the test method. ${ }^{6}$ The effect of fatigue and potentiation may not be desirable in isokinetic measurements. 
The ability to control these factors could enhance the reliability and validity of the isokinetic data. Research examining the differences between the two methods of testing concentric and eccentric muscle performance are not currently available in the literature.

The reliability of isokinetic measurements is an important consideration when evaluating data in the clinical/research setting. Several researchers have demonstrated good reliability when assessing the concentric muscle performance of the shoulder. ${ }^{2,13,16}$ Only a few reports have described the reliability of measurement protocols that include eccentric performance of the shoulder. ${ }^{5,8,10}$

The purpose of our investigation was to compare torque production of the shoulder rotators when using an interrupted versus a continuous isokinetic test protocol. A subproblem examined was the reliability of torque measurements obtained with the test protocols.

\section{METHODS}

Subjects

Twenty-eight women (age $20 \pm 1.5$ years; weight $58.4 \pm 6.4 \mathrm{~kg}$; height $162 \pm 5.6 \mathrm{~cm}$ ) volunteered to participate in this investigation. Dominant, injury-free upper extremities were assessed. Upper extremity dominance was determined by asking subjects which arm they used to perform gross motor activities, such as throwing a ball. Subjects had no previous experience with isokinetic testing of the shoulder. Subjects were informed of the procedure and inherent risks of the investigation and signed an informed consent form in accordance with institutional human investigation committee guidelines. Subjects were randomly assigned to participate in either a continuous or interrupted stroke protocol before the testing procedures.

\section{Measuring Device}

The Kin-Corn was used to measure maximal voluntary concentric and eccentric contractions of the internal and external rotators of the dominant shoulder. The dynamometer was calibrated internally before each testing session. Additional Kin-Corn accessories used in this investigation were the movable chair, Velcro straps, shin pad adapter for the load cell, and the V support.

\section{Procedures}

\section{General Warm-Up}

Before isokinetic measurement, subjects participated in a general warm-up of the shoulder, using general shoulder flexibility exercises. Subjects were instructed to complete five to 10 repetitions of each exercise before and after testing. The test instructions, supervision of warm-up, and testing were provided by the test administrator.

\section{Subject Positioning}

Each subject was provided with a brief orientation to the Kin-Corn testing system after the general shoulder warm-up exercises. Subjects were positioned sitting at a right angle to the dynamometer to allow testing to be performed with the shoulder in the frontal plane. The dynamometer was angled to 45 degrees (to accommodate 45 degrees of glenohumeral abduction) and set at the lowest height available. The axis of glenohumeral rotation was aligned with the axis of the lever arm by varying the height of the seat. The best possible alignment was determined through visual inspection in the frontal and saggital planes, and the seat height was 
recorded for each subject. The lower extremities of each subject were supported on a chair throughout the testing procedure.

Trunk and hip stabilization was used during the warm-up and testing repetitions. Stabilization of the trunk was provided via a Velcro strap placed horizontally across the chest and secured to the back of the chair. Hip stabilization was provided via a Velcro strap positioned approximately 45 degrees across the pelvis and secured to the chair. The movable chair was locked in place once the optimal test position was obtained.

The elbow was positioned in approximately 90 degrees of flexion. The elbow/shoulder was supported by the use of the $\mathrm{V}$ pad. The inverted load cell pad was positioned with the axis of the pad placed approximately $2 \mathrm{~cm}$ proximal to the wrist joint. The length of the lever arm was entered into the computer. Average torque values were measured from 20 degrees internal rotation to 90 degrees external rotation for all subjects. Neutral or 0 degrees of rotation was determined by the test administrator, with the assistance of a carpenter's level on the lever arm when the forearm was in the horizontal position. Mechanical range of motion blocks were set at approximately 100 degrees of external rotation and 40 degrees of internal rotation.

\section{Test Procedures}

Warm-up contractions preceded the test of maximal effort. The warm-up consisted of five repetitions, which included three submaximal and two maximal concentric and eccentric contractions specific to the test protocol (continuous versus interrupted). A 2-minute rest interval was provided between warm-up and test repetitions.

The testing procedure $\mathrm{c}$ insisted of three repetitions of maximal voluntary concentric and eccentric contractions of the internal and external rotators at test velocities of 60 and 180 $\mathrm{deg} / \mathrm{sec}$. Each maximal concentric contraction was measured first, followed by an eccentric contraction. The order of testing the internal and external rotators was counterbalanced for each subject. There was a 2-minute rest between testing of the muscle groups. Each subject was tested at 60 followed by $180 \mathrm{deg} / \mathrm{sec}$. A 2-minute rest was provided between testing velocities. During tests of maximal contractions, subjects were not allowed to use their nondominant upper extremity to provide stabilization.

The interrupted stroke protocol group was allowed a 5-second rest between concentric and eccentric contractions. The continuous protocol group was not allowed a rest interval between concentric/eccentric action or between the three test cycles.

Subjects participated in an initial test (practice) session after the exact testing procedure described above. The practice session was designed to familiarize each individual with the test procedures and facilitate accommodation to the eccentric mode of testing. The test session was 2 to 3 days after the practice session, using the exact procedure as outlined above. All of the participants from each test protocol group returned 5 to 7 days to be retested to assess test-retest reliability. No effort was made to control the activity of subjects between test sessions.

\section{Test Parameters}


The gravity correction option was implemented to compensate for the effects of gravity for all test conditions. The upper extremity was placed within the range of motion to be tested (20 degrees internal rotation to 90 degrees external rotation) in the horizontal position ( 0 degrees). Each subject was asked to relax the upper extremity and allow the Kin-Com to measure the force due to gravity. This procedure was implemented before each session. Variation within subject trials was within $1 \mathrm{Nm}$. The preload force was $25 \mathrm{~N}$ across all test conditions.

Verbal encouragement was not used during warm-up or test repetitions. Verbal encouragement was omitted because of the difficulty in providing consistent feedback across subjects and test sessions. Subjects were not provided visual feedback during warm-up or during test repetitions.

\section{Data Analysis}

Average torque values for each subject were measured from 20 degrees of internal rotation to 90 degrees of external rotation. Average torque values for each subject were derived from the average of three maximal voluntary contractions across each level of the within-subjects variables (test speed, mode of contraction, and muscle group). All torque values in Nm were obtained from the Kin-Corn results program.

To describe the difference in average torque values between mode of contraction, test speed, and test protocol, a mixed model (one between, two within) analysis of variance (ANOVA) was used to analyze data from test session 1 for the internal and external rotators. The MANOVA program from the Statistical Package for the Social Sciences ${ }^{11}$ was used to compile the one between, two within design analysis. The $\alpha$ level for all tests of statistical significance was established as 0.05 .

To determine the test-retest reliability of the isokinetic measurements within this sample, intraclass correlation coefficients (ICCs) were calculated for subjects across test sessions 1 and 2 according to Shrout and Fleiss. ${ }^{15}$ The ICC equation selected was $(2,3)$ to allow generalization of the findings and because the measure of interest was comprised of an average of three measurements. All ICCs were computed using the ICC.BAS basic program. ${ }^{3}$

To describe the precision of measurement, Standard Error of Measurement (SEM) estimates were computed for the test-retest. Unlike the ICC, the SEM possesses the unit of measure and provides the opportunity to calculate a range in which the subject's true score is located. SEMs were calculated by multiplying the standard deviation of the measurements by the square root of 1 minus the reliability coefficient.

\section{RESULTS}

The means and standard deviations for average torque of the shoulder external and internal rotators are presented in Figures 1 and 2. The average external rotator torque values of the continuous group were not significantly $(\mathrm{p}=0.62)$ different from the interrupted group. The mean 


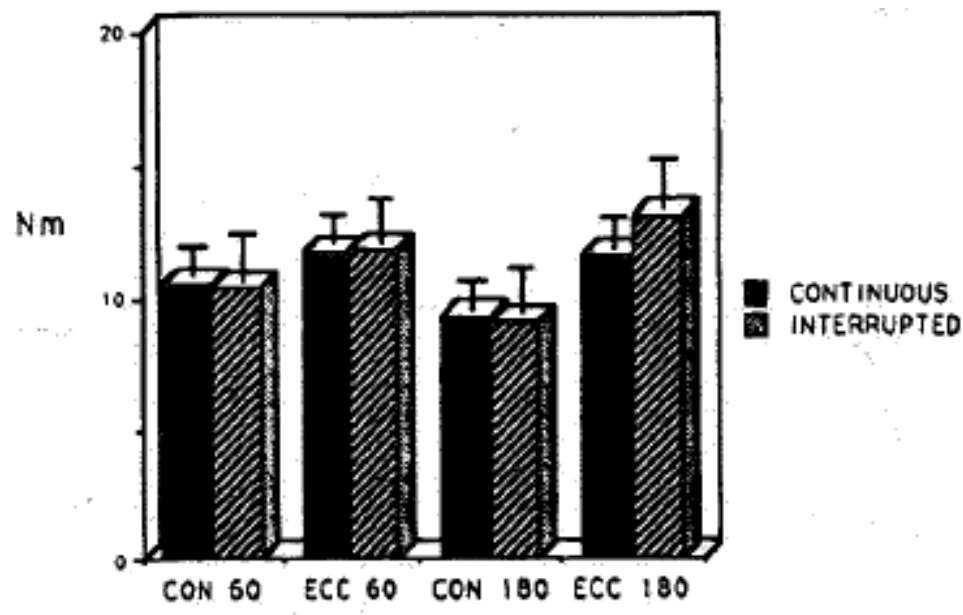

Figure 1 Means and standard deviations for average torque of the external rotators.

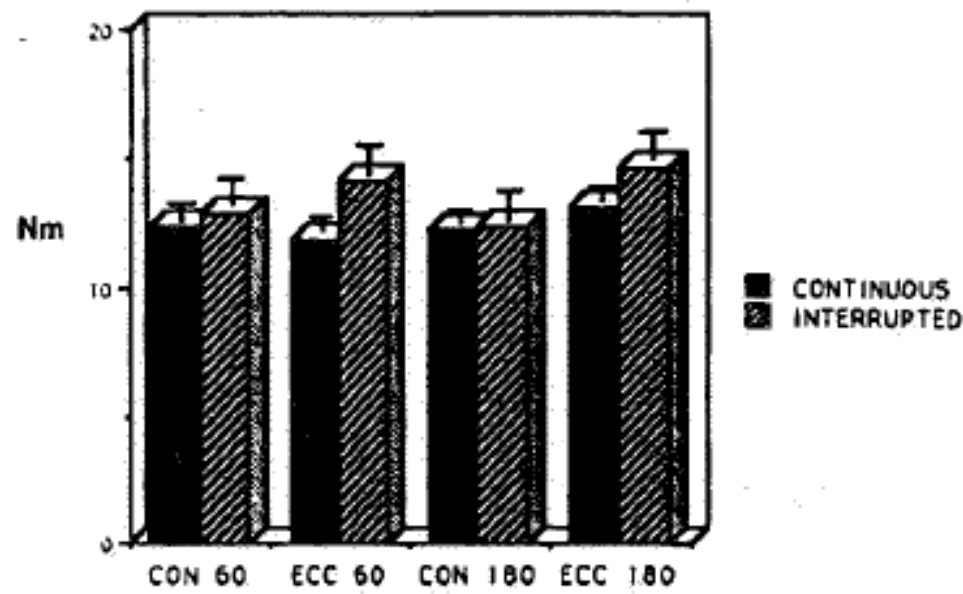

Figure 2 Means and standard deviations for average torque of the internal rotators. 
Table 1 Reliability estimates for test-retest reliability of average torque values of the external rotators (SEM in Newton-meters).

\begin{tabular}{cllll}
\hline Protocol & CON 60 & ECC 60 & CON 180 & ECC 180 \\
\hline Continuous & & & & \\
ICC & 0.79 & 0.72 & 0.82 & 0.57 \\
SEM* & 0.9 & 1.3 & 0.7 & 1.5 \\
Interrupted & & & & \\
ICC & 0.93 & 0.89 & 0.83 & 0.91 \\
SEM $^{*}$ & 0.5 & 1.0 & 0.7 & 0.9 \\
\hline
\end{tabular}

CON, concentric; ECC, eccentric; ICC, intraclass correlation coefficient; SEM, standard error of measurement.

*Values are Newton-meters.

Table 2 Reliability estimates for test-retest reliability of average torque values of the internal rotators.

\begin{tabular}{cllll}
\hline Protocol & CON 60 & ECC 60 & CON 180 & ECC 180 \\
\hline Continuous & & & & \\
ICC & 0.88 & 0.84 & 0.84 & 0.88 \\
SEM* & 1.1 & 1.3 & 1.2 & 1.2 \\
Interrupted & & & & \\
ICC & 0.92 & 0.91 & 0.94 & 0.94 \\
SEM $^{*}$ & 0.9 & 1.4 & 0.8 & 1.2 \\
\hline
\end{tabular}

CON, concentric; ECC, eccentric; ICC, intraclass correlation coefficient; SEM, standard error of measurement.

*Values are Newton-meters.

average internal rotator torque values of the continuous group were not significantly $(\mathrm{p}=0.38)$ different from the interrupted group.

\section{Estimates of Test-Retest Reliability}

Comparisons of ICCs are presented in Tables 1 and 2. ICCs calculated for mean average torque of the external rotators ranged from 0.57 to 0.82 for the continuous protocol and from 0.83 to 0.93 for the interrupted protocol. ICCs calculated for average torque of the internal rotators ranged from 0.84 to 0.88 for the continuous protocol and from 0.91 to 0.94 for the interrupted protocol.

Comparisons of SEMs are presented in Tables 1 and 2. SEMs calculated for average torque of the external rotators ranged from 0.7 to $1.5 \mathrm{Nm}$ for the continuous protocol and from 0.5 to 1.0 $\mathrm{Nm}$ for the interrupted protocol. SEMs calculated for the average torque of the internal rotators ranged from 1.1 to $1.3 \mathrm{Nm}$ for the continuous protocol and from 0.8 to $1.4 \mathrm{Nm}$ for the interrupted protocol.

\section{DISCUSSION}


Our investigation found no significant differences in average torque production of the shoulder internal and external rotator muscle groups between continuous and interrupted test protocols. This finding contrasts with that of Stratford et al., ${ }^{17}$ who reported that peak torque values for the knee flexors and extensors obtained with an interrupted protocol were 5\% greater than those obtained with a continuous protocol when using a Cybex dynamometer (Cybex, Ronkonkoma, NY). The difference was attributed to a decline in the torque production over the five trials with the continuous protocol, suggesting a fatigue factor.

The placement of rest intervals between concentric/ eccentric contractions or between concentric/eccentric cycles may affect torque production. Barnes' suggested that an intertrial rest interval allows the muscle to recover and could influence torque production when compared with continuous contractions. Stratford et al.$^{17}$ attributed increased torque production between protocols to a 30-second rest interval placed between concentric knee extension/flexion cycles.

A decline in torque production has not been reported by researchers using the continuous mode of testing to assess concentric and eccentric performance of the knees and shoulder. ${ }^{10}$ Kramer $^{9}$ reported only that subjective reports indicated that fatigue was not a factor. $\mathrm{Ng}$ and Kramer ${ }^{10}$ found no significant $(\mathrm{p}<0.05)$ differences in the three highest of four repetitions when testing the concentric and eccentric performance of the shoulder rotators. However, their elimination of the lowest peak torque may have masked any trends that were present within the four contractions.

A 5-second rest interval between concentric and eccentric contractions has been reported in the literature in investigations using the Kin-Com to assess the musculature of the knee. ${ }^{18}{ }^{29}$ The effect of fatigue was not reported by either researcher. The 5-second rest interval used in our investigation may not have been adequate to discriminate between test protocols. An increase in the duration of the rest interval may result in an increase in overall torque production secondary to a reduction in fatigue over trials.

The effect of a previous contraction on a subsequent contraction may also impact torque production and contribute to variation between trials. Tredinnick and Duncan ${ }^{18}$ used a 5-second rest between contractions to minimize the effects of a concentric contraction on a subsequent eccentric contraction. Kramer ${ }^{9}$ reported that peak torque of the first concentric contraction was frequently lower than the two that followed, resulting in the analysis of data being limited to the two highest torque patterns.

The limited interaction between continuous concentric and eccentric contractions may be explained by the inclusion of an isometric preload of $25 \mathrm{~N}$ placed between contractions. The subject must generate this preset force to initiate movement. Helgeson and Gajdosik ${ }^{7}$ reported that peak torque of the knee extensors was increased after both an eccentric and isometric preload. No significant difference between these two precontraction conditions was detected. Researchers must report if and what preload is used for data collection due to its impact on torque production.

\section{Estimates of Reliability}


ICCs were greater for the interrupted protocol when compared with the continuous protocol. All ICCs were greater than 0.8 , with the exception of those obtained from the continuous protocol for the external rotators. The overall magnitude of these coefficients reflect good reliability.

The range of test-retest reliability coefficients obtained within the interrupted protocol for this sample were consistent with reports of reliability coefficients obtained when assessing concentric and eccentric performance of the shoulder. ${ }^{5,9} 1 \mathrm{CCs}$ calculated for the continuous protocol were within this range for the internal rotators, although estimates were lower for the external rotators. Hageman et al..$^{5}$ reported Pearson correlation coefficients ranging from 0.83 to 0.93 , and Hellwig and Perrin reported ICCs ranging from 0.76 to 0.94 .

The interrupted protocol has a greater or equal precision of measurement than does the continuous protocol except for eccentric performance of the internal rotators at $60 \mathrm{deg} / \mathrm{sec}$. However, the difference $(0.1)$ between protocols for the internal rotators at $60 \mathrm{deg} / \mathrm{sec}$ was negligible in terms of clinical importance. Within the continuous protocol, measurement of eccentric muscle performance appears to be less precise than the measurement of concentric performance.

Lower estimates of reliability found with the continuous protocol may be a function of stabilization of the upper quarter during testing. An empirical observation during testing subjects with the continuous test protocol was the presence of increased substitution of the upper quarter. The cross chest strap provided adequate control for subjects tested in the interrupted mode. However, it did not appear to control extraneous movements during the continuous test sequence. A suggestion to facilitate upper quarter stabilization with the Kin-Corn would be to incorporate a stabilization strap that crosses the anterior aspect of the shoulder joint as reported by Ng and Kramer, ${ }^{9}$ They did not assess test-retest reliability, but reported good intertrial reliability using a continuous assessment method for the shoulder.

\section{Suggestions for Further Study}

Continued investigation is necessary to assist in defining the role of isokinetics in clinical practice and research. To facilitate the use of isokinetic measures, the impact of test variables on torque production and reliability need to be established. Avenues for potential study include the length of rest intervals and the effect of preload on test sequence.

\section{Acknowledgment}

We acknowledge the assistance of Joe H. Gieck, EdD, PT, ATC, Ethan Saliba, PhD, PT, ATC, Donald Ball, EdD, and Stephen Sprigle, $\mathrm{PhD}$, for their assistance in the organization and implementation of this study.

\section{REFERENCES}

1. Barnes WS: Isokinetic fatigue curves at different contractile velocities. Arch Phys Med Rehabil 62:66-69, 1981.

2. Day RW, Moore RT, Patterson P: Isolcinetic torque production of the shoulder in a functional movement pattern. Athl Train 3:333-337, 1988.

3. Delitto A, Strube M J: Reliability in the clinical setting. Research Section Newsletter:APTA 24:2-8, 1991. 
4. Griffin JW: Differences in elbow flexion torque measured concentrically, eccentrically and isometrically. Phys Ther 67:1205-1208, 1987.

5. Hageman PA, Mason DK, Rydlund KW, et al.: Effects of position and speed on eccentric and concentric isokinetic testing of the shoulder rotators. J Orthop Sports Phys Ther 11:6469, 1989.

6. Harding B, Black T, Bruulsema A, et al.: Reliability of a reciprocal test protocol performed on the Kinetic Communicator: an isokinetic test of knee extensor and flexor strength. $\mathrm{J}$ Orthop Sports Phys Ther 10:218-223, 1988.

7. Helgeson K, Gajdosik R: The stretch shortening cycle of the quadriceps femoris muscle group measured by isokinetic dynamometry. J Orthop Sports Phys Ther 17:17-23, 1993.

8. Hellwig EV, Perrin DH: A comparison of two positions for assessing shoulder rotator peak torque: the traditional frontal plane versus the plane of the scapula. Isokinet Exerc Sci 1:202206, 1991.

9. Kramer IF: Reliability of knee extensor and flexor torques during continuous concentriceccentric cycles. Arch Phys Med Rehabil 71:460-464, 1990.

10. Ng LR, Kramer JS: Shoulder rotator torques in female tennis and nontennis players. J Orthop Sports Phys Ther 13:40-46, 1991.

11.Norusis, MJ: SPSSX Advanced Statistics Guide. Chicago,IL: SPSS Inc., 1988.

12.Patton RW, Hinson MM, Arnold BR, et al.: Fatigue curves of isokinetic contractions. Arch Phys Med Rehabil 59:507509, 1978.

13.Perrin DH: Reliability of isokinetic measures. Athletic Training 21:319-321, 1986.

14.Perrin DH, Hellwig EV, Tis LL, et al.: Effect of gravity correction on shoulder rotation isokinetic average force and reciprocal muscle group ratios. Isokinet Exerc Sci 2:3033, 1992.

15. Shrout PE, Reiss JL: Intraclass correlations: uses in assessing rater reliability. Psychol Bull 86:420-428, 1979.

16. Soderberg GJ, Blaschak MJ: Shoulder internal and external rotation peak torque production through a velocity spectrum in differing positions. J Orthop Sports Phys Ther 8:518 — 524, 1987.

17. Stratford PW, Bruulsema A, Maxwell B, et al.: The effect of inter-trial rest interval on the assessment of isokinetic thigh muscle torque. I Orthop Sports Phys Ther 11:362366, 1990.

18. Tredinnick TJ, Duncan PW: Reliability of measurements of concentric and eccentric isokinetic loading. Phys Ther 68:656-659, 1988.

19. Trudelle-Jackson E, Meske N, Highgenboten C, et al.: Eccentric/concentric torque deficits in the quadriceps muscle. J Orthop Sports Phys Ther 11:142-145, 1989.

20. Wilhite MR, Cohen ER, Wilhite SC: Reliability of concentric and eccentric measurements of quadriceps performance using the Kin Corn dynamometer: the effect of testing order for three different speeds. J Orthop Sports Phys Ther 15:175-182, 1992. 